\title{
Prototype single-balloon enteroscopy with passive bending and high force transmission improves depth of insertion in the small intestine
}

\author{
Yasuhiro Morita $^{1 *}$, Shigeki Bamba ${ }^{2 *}$, Osamu Inatomi ${ }^{1}$, Kenichiro Takahashi $^{1}$, Takayuki Imai ${ }^{1}$, Masaki Murata ${ }^{1}$, \\ Masashi Ohno ${ }^{1}$, Masaya Sasaki ${ }^{2}$, Tomoyuki Tsujikawa ${ }^{3}$, Akira Andoh ${ }^{1}$ \\ ${ }^{1}$ Department of Medicine, ${ }^{2}$ Division of Clinical Nutrition, and ${ }^{3}$ Department of Comprehensive Internal Medicine, Shiga University of Medical \\ Science, Otsu, Japan
}

Background/Aims: We retrospectively analyzed Crohn's disease (CD) patients with small intestinal strictures who underwent single-balloon enteroscopy (SBE) to ascertain whether prototype SBEs with a passive bending mechanism and high force transmission insertion tube had better insertability in the small intestine than a conventional SBE. Methods: Among 253 CD patients who underwent SBE, we identified 94 CD patients who had undergone attempted endoscopic balloon dilatation (EBD) for small intestinal stenosis for inclusion in this study. We analyzed whether the type of scope used for their initial procedure affected the cumulative surgery-free rate. For the insertability analysis, patients who underwent SBE at least twice were divided into 3 groups according to the type of scope used: conventional SBE only, prototype SBE only, and both conventional and prototype SBEs. For each group, depth of insertion, procedure time, and number of EBDs were compared in the same patient at different time points. Results: The success rate of EBD was 88.3\%. The 5- and 10-year cumulative surgery-free rate was $75.7 \%$ and $72.8 \%$, respectively. Cox regression analysis indicated that the factors contributing to surgery were long stricture $(\geq 2 \mathrm{~cm})$, EBD failure, and elevated Crohn's Disease Activity Index, but not the type of scope used for EBD. The prototype SBEs significantly improved the depth of insertion $(P=0.03$, Wilcoxon's signed-rank test). Conclusions: In CD patients with small intestinal stenosis, the prototype SBEs with a passive bending mechanism and high force transmission insertion tube did not improve long-term EBD outcome but did improve deep insertability. (Clinical Trial Registration No. UMIN000037102) (Intest Res 2020;18:229-237)

Key Words: Balloon-assisted enteroscopy; Dilation; Double-balloon enteroscopy

\section{INTRODUCTION}

Crohn's disease (CD) is a chronic inflammatory intestinal disorder that involves transmural inflammation of the GI tract and can potentially cause stricture and fistula formation. ${ }^{1}$ Stricture in the small intestine is one of the most common intestinal complications and sometimes requires intestinal re-

Received November 18, 2019. Revised December 25, 2019.

Accepted January 13, 2020.

Correspondence to Shigeki Bamba, Division of Clinical Nutrition, Shiga University of Medical Science, Seta-Tsukinowa, Otsu 520-2192, Japan. Tel:

+81-77-548-2899 Fax:+81-77-548-2499 E-mail: sb@belle.shiga-med.ac.jp

*These authors contributed equally to this study. section. The cumulative surgery rate after diagnosis of CD is reported to be $46 \%-62 \%$ at 5 years and $61 \%-75 \%$ at 10 years. $^{2-4}$ In addition, a greater number of strictureplasties is associated with a higher the risk of reoperation. ${ }^{5}$ To avoid the risk of short bowel syndrome, intestinal resection should be kept to a minimum. $^{6}$

Endoscopic balloon dilatation (EBD) has been shown to be an effective treatment that helps to avoid surgery, ${ }^{7-10}$ and the Japanese clinical practice guidelines for IBD recommend EBD when obstructive symptoms do not improve with drug therapy alone. ${ }^{11}$ Lian et al. ${ }^{12}$ reported that EBD could delay surgery for 6.45 years in a study of 176 EBD-treated and 131 surgically 
treated CD patients. Although it is reported that EBD failure, strictures with fistula, long stricture, and multiple stenoses are factors that contribute to intestinal resection in CD patients with stenosis in the small intestine ${ }^{13-17}$ there are few reports about the long-term outcomes of EBD in CD patients with strictures in the small intestine.

We were given the opportunity to use prototype single-balloon enteroscopes (SBEs) with a passive bending mechanism and high force transmission insertion tube. Colonoscopes with this mechanism and insertion tube have been reported to reduce the cecal intubation time and pain during the procedure. ${ }^{18,19}$ In a prospective study of 60 patients, the use of a prototype SBE with a passive bending mechanism and high force transmission insertion tube was found to shorten of terminal ileum intubation time compared with use of the SIF-Q260, a conventional SBE. ${ }^{19}$ However, in that study, the SBE was inserted only $20 \mathrm{~cm}$ from the ileocecal valve, and the results mainly reflect improvement of insertability in the colon.

We conducted this retrospective study to test our hypothesis that the prototype SBEs with a passive bending mechanism and high force transmission insertion tube can be inserted deeper than a conventional SBE in the small intestine in patients with stricturing $\mathrm{CD}$ who required $\mathrm{EBD}$. We also analyzed whether the type of scope used affects the long-term outcomes of EBD.

\section{METHODS}

\section{Ethical Considerations}

This retrospective study was approved by the Ethics Committee of Shiga University of Medical Science (approval No. R2019121) and informed consent was waived. The study is registered in the University Hospital Medical Network Clinical Trials Registry (UMIN000037102).

\section{Patients}

Among the $253 \mathrm{CD}$ patients who underwent SBE at Shiga University of Medical Science from October 2005 to December 2017, we identified 94 who had undergone attempted EBD for small intestinal stenosis for inclusion in this study (Fig. 1). The 94 CD patients included 11 patients with unsuccessful EBD with failed dilation of stenosis. Disease phenotype and status was defined according to the Montreal classification. ${ }^{20}$

\section{Scopes and Procedures}

Four scopes were used in this study: the SIF-Q260 (Olympus,

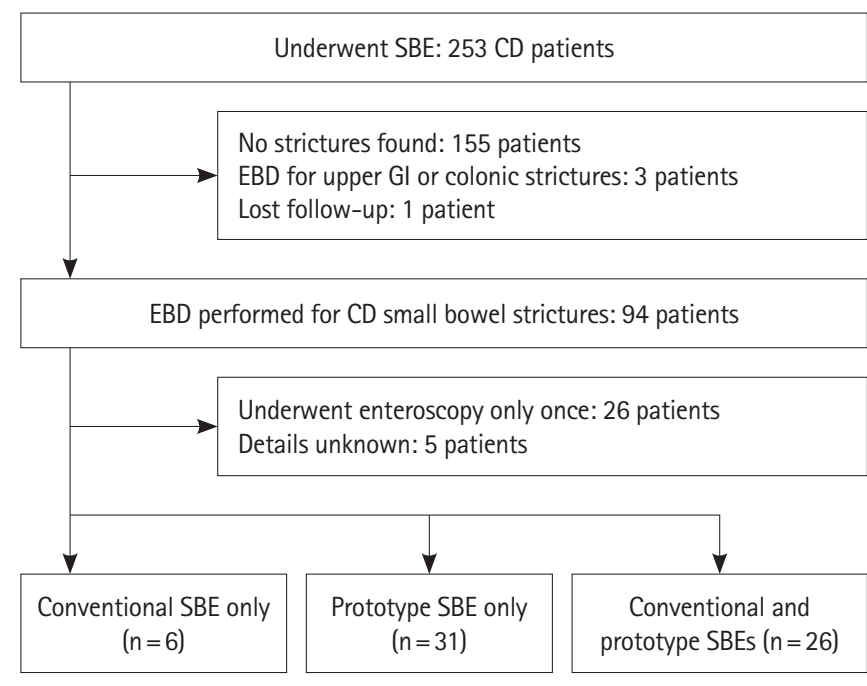

Fig. 1. Study participants. Among $253 \mathrm{CD}$ patients who underwent single-balloon enteroscopy (SBE), 94 underwent attempted endoscopic balloon dilatation (EBD) for small intestinal stenosis. The clinical characteristics of these 94 patients are shown in Table 1. To compare the insertability, patients who underwent SBE at least twice during the observation period were extracted and divided into 3 groups: conventional SBE only, prototype SBE only, and both conventional and prototype SBEs. Detailed information of these groups is shown in Table 3.

Tokyo, Japan), which is a conventional SBE that does not have a passive bending mechanism or high force transmission insertion tube, and the SIF-Y0002, SIF-Y0007, and SIF-Y0013 (Olympus), which are prototype SBEs that have a passive bending mechanism and high force transmission insertion tube. The specifications of the scopes are shown in Supplementary Table 1. Briefly, SIF-Y0002 is a model based on SIFQ260 with addition of a passive bending mechanism and high force transmission insertion tube. SIF-Y0007 is a model based on SIF-Y0002 with addition of a magnifying mechanism. SIFY0013 is a model designed for the 290 series light source and is equipped with a passive bending mechanism, a high force transmission insertion tube, and an instrumental channel of $3.2 \mathrm{~mm}$ in diameter.

The endoscope was selected by the endoscopist. If both conventional and prototype scopes could be used, the prototype SBE was often selected. However, if there were multiple endoscopic examinations on the same day, they were used alternately by adjusting the cleaning time. All endoscopic procedures were performed by 3 experienced endoscopists (T.T., S.B., and K.T.).

EBD was performed using a through-the-scope balloon catheter $\left(\mathrm{CRE}^{\mathrm{TM}}\right.$ balloon dilatation catheter; Boston Scientific 
Co., Natick, MA, USA). In principle, the dilatation balloon was inflated to at least $15 \mathrm{~mm}$ and maintained for 30 seconds to 2 minutes. Carbon dioxide insufflation was performed during all procedures. EBD was performed under conscious sedation with midazolam or propofol.

We defined successful EBD as confirmation of dilatation of the stenosis under fluoroscopy. In patients with multiple small intestinal strictures, we defined successful EBD as confirmed dilatation of the stenosis responsible for the obstructive symptoms. Indications for EBD using SBE for small intestinal strictures at our institution include the following: no fistula or abscess in the stenosis and no untreated deep ulcer. At our institution, SBE is performed under fluoroscopic guidance to confirm the length and number of stenoses. If we find any asymptomatic stenosis that prevents passage of the scope, we perform EBD on that stenosis. Patients were hospitalized until the day after EBD and were discharged after confirming there were no symptoms such as hemorrhage and abdominal pain on the day after EBD. Procedure-related complications were defined as intestinal perforation and active bleeding requiring surgery or blood transfusion.

\section{Long-term Outcome}

The long-term outcome was determined as the cumulative surgery-free rate after initial EBD. Surgery was performed for strictures that were not resolved by medical or endoscopic therapy, or for intestinal perforation as a complication of EBD. We examined whether long-term outcome was influenced by clinical background, including stenotic status, blood biochemistry, and the scope initially used. The analysis of long-term outcome included patients who were observed for at least 3 weeks after the initial EBD.

\section{Insertability and Scope Used}

To investigate the usefulness of deep insertion of prototype SBEs with a passive bending mechanism and high force transmission insertion tube, we selected cases in which 2 or more SBE procedures were performed during the follow-up period. We then divided these cases into 3 groups according to the scope used: (1) the conventional SBE only, (2) a prototype SBE only, and (3) both conventional and prototype SBEs. For comparison of deep insertability in the small intestine, the 2 most recent procedures were compared in each group. When both the conventional and prototype SBEs were used, the most recent switching opportunity was compared. During all procedures, depth of small intestinal insertion was estimated using the method described by May et al. ${ }^{21}$ The procedure time was defined as starting insertion of the endoscope to finishing removal of the endoscope from the patient.

\section{Statistical Analyses}

Cumulative surgery-free rates were analyzed using the Kaplan-Meier method and log-rank test. In addition, factors contributing to surgery were examined using Cox regression anal-

Table 1. Clinical Background of the Patients

\begin{tabular}{|c|c|}
\hline Characteristic & Value $(n=94)$ \\
\hline Sex (male/female) & $77 / 17$ \\
\hline Age at diagnosis (yr) & $28.8 \pm 13.0$ \\
\hline Age at first EBD (yr) & $37.0 \pm 12.4$ \\
\hline BMI $\left(\mathrm{kg} / \mathrm{m}^{2}\right)$ & $21.3 \pm 3.3$ \\
\hline Smoking (yes/no) & $13 / 81$ \\
\hline Age at diagnosis (A1/A2/A3) & $8 / 72 / 14$ \\
\hline Disease phenotype at first EBD (B1/B2/B3) & $16 / 62 / 16$ \\
\hline Disease location at first EBD (L1/L3) & $52 / 42$ \\
\hline Perianal lesion present (yes/no) & $30 / 64$ \\
\hline History of surgery (yes/no) & $48 / 46$ \\
\hline Success of EBD (yes/no) & $83 / 11$ \\
\hline Symptoms of obstruction before EBD (yes/no) & $71 / 23$ \\
\hline Serum albumin (g/dL) & $3.8 \pm 0.5$ \\
\hline Serum CRP (mg/dL) & $0.6 \pm 1.0$ \\
\hline CDAl at first EBD (point) & $127.3 \pm 76.8$ \\
\hline \multicolumn{2}{|l|}{ Concomitant treatments } \\
\hline Thiopurine (yes/no) & $29 / 65$ \\
\hline Anti-TNF- $\alpha$ antibody (yes/no) & $43 / 51$ \\
\hline \multicolumn{2}{|l|}{ Scope used for initial EBD } \\
\hline Conventional SBE & 44 \\
\hline Prototype SBE & 50 \\
\hline \multicolumn{2}{|l|}{ Stricture site } \\
\hline Single $(\%)$ & 49 \\
\hline Multiple (\%) & 45 \\
\hline 2 & 22 \\
\hline 3 & 11 \\
\hline$\geq 4$ & 12 \\
\hline De novo type of strictures (yes/no) & $77 / 17$ \\
\hline Stricture length (cm) & $1.3 \pm 0.9$ \\
\hline Long stricture ( $\geq 2 \mathrm{~cm}$, yes/no) & $26 / 68$ \\
\hline
\end{tabular}

Values are presented as mean \pm SD.

$E B D$, endoscopic balloon dilatation; $A 1, \leq 16$ years; $A 2,17-40$ years; $A 3$, $>40$ years; $B 1$, non-stricturing, non-penetrating; $B 2$, stricturing; $B 3$, penetrating; L1, ileal; L3, ileocolonic; SBE, single-balloon enteroscopy. 
ysis. All variables with $P$-values less than 0.15 in univariate analysis were entered into multivariate analysis. We also included sex, age, and other factors previously described. ${ }^{13}$ Differences in insertability were analyzed using Wilcoxon's signedrank test. Statistical significance was defined as $P<0.05$. GraphPad Prism 8.0.0 (GraphPad, San Diego, CA, USA) and SPSS version 25.0 (IBM Corp., Armonk, NY, USA) were used for statistical analysis.

\section{RESULTS}

A total of $94 \mathrm{CD}$ patients with a mean follow-up period of 1,886 days were included in this study. Baseline characteristics at initial EBD are shown in Table 1. The mean number of EBDs performed per patient during the follow-up period was 3.3, and the mean interval between the initial EBD and the second EBD was 508 days. Among 83 patients who underwent successful EBD, 80 (96.4\%) were initially dilated to more than $15 \mathrm{~mm}$. Of the remaining 3 patients, 1 was initially dilated to $12 \mathrm{~mm}$ and 2 were initially dilated to $10 \mathrm{~mm}$.
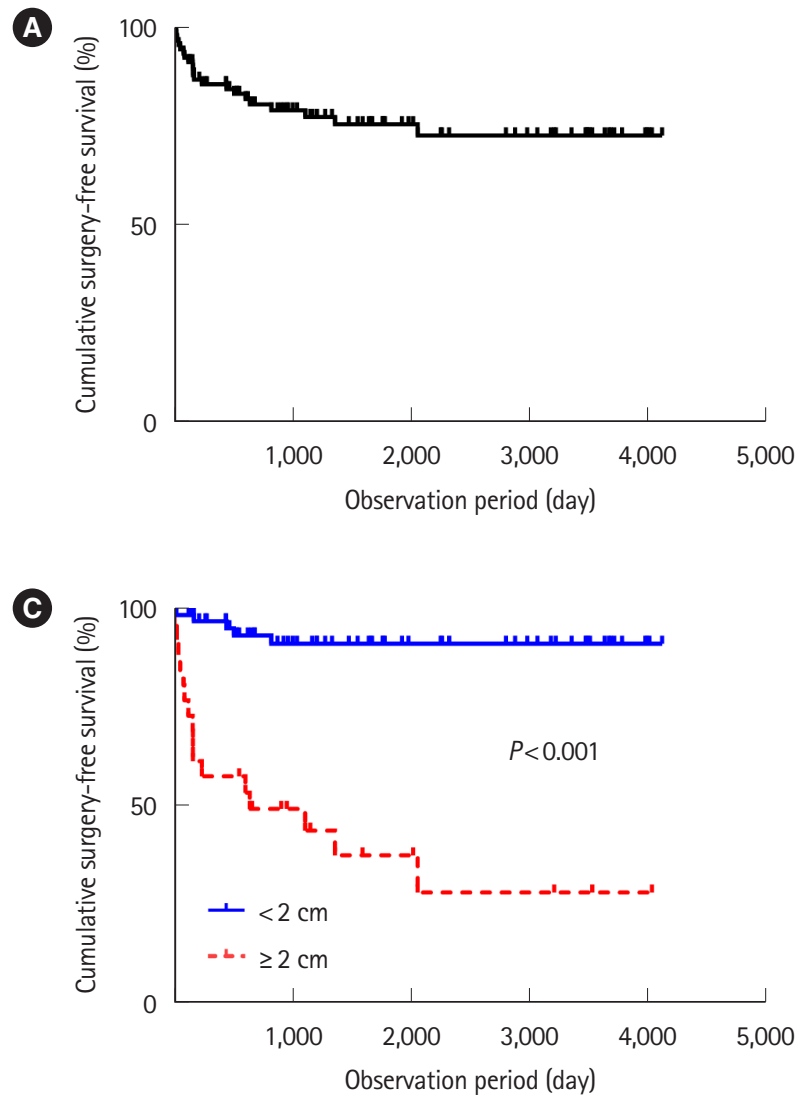

The EBD success rate was $88.3 \%$ (83/94). The 5- and 10-year cumulative surgery-free rate was $75.7 \%$ and $72.8 \%$, respectively (Fig. 2A). Table 2 shows the results of multivariate analysis of factors contributing to intestinal resection. Elevated CDAI, long stenosis ( $\geq 2 \mathrm{~cm}$ ), and EBD failure were identified as factors contributing to intestinal resection. The results were also confirmed by Kaplan-Meier analysis (Fig. 2B-D). Factors such as prototype scope used for initial EBD, number of stenoses, and de novo stenosis were not associated with intestinal resection. In addition, the risk of surgery increased by 2.53 times when the length of stenosis increased by $1 \mathrm{~cm}$ in a successful EBD case (HR, 2.53; 95\% CI, 1.580-4.069; $P<0.001$ ).

During the follow-up period, 314 EBDs were performed in 94 CD patients, and 4 patients $(1.29 \%, 4 / 314)$ had intestinal perforation as a complication. There were no cases of bleeding requiring surgery or blood transfusion.

The insertability and the scope used were compared between the groups shown in Table 3. We compared depth of insertion, procedure time, and number of EBDs between 2 procedures in the same patient at different times (Fig. 3). Com-
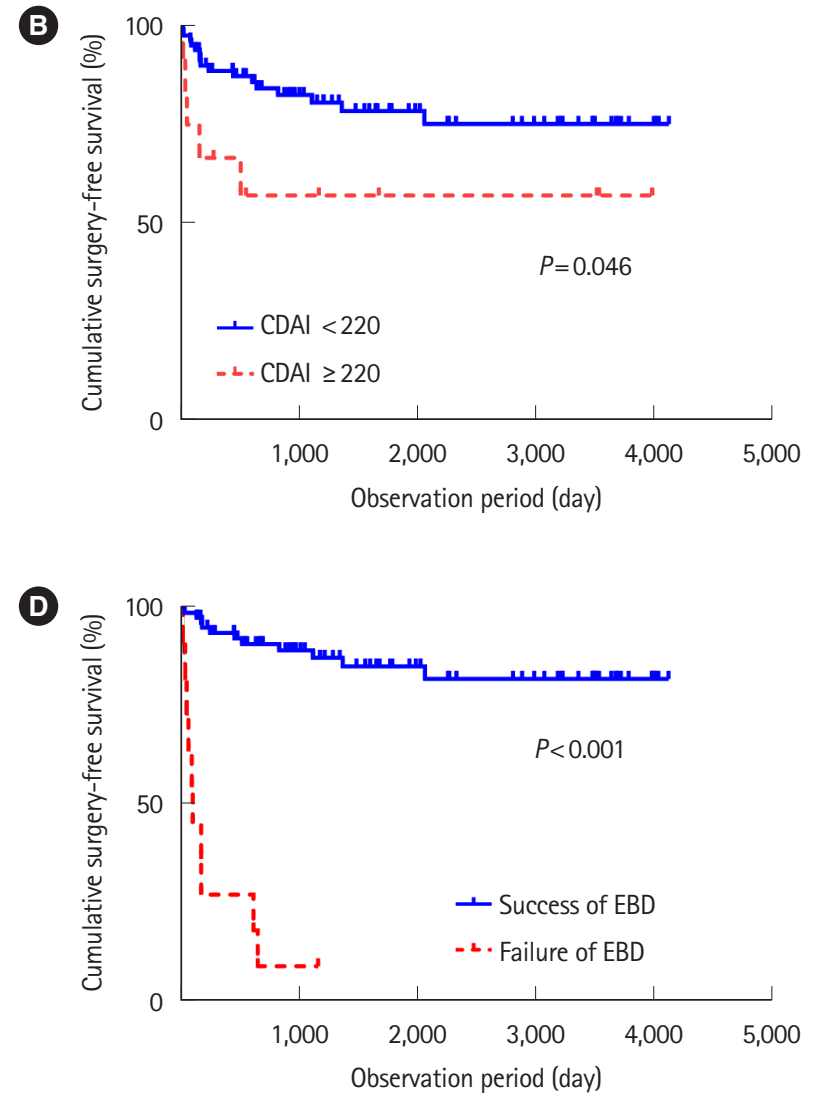

Fig. 2. Cumulative surgery-free survival rate. Kaplan-Meier curves depicting cumulative surgery-free survival for all patients (A), and stratified by CDAI (B), stricture length (C), and endoscopic balloon dilatation (EBD) success (D). P-values for each curve were calculated using the log-rank test. 
Table 2. Factors Associated with Intestinal Resection for all CD Patients

\begin{tabular}{|c|c|c|c|c|}
\hline \multirow{2}{*}{ Factor } & \multicolumn{2}{|c|}{ Univariate analysis } & \multicolumn{2}{|c|}{ Multivariate analysis } \\
\hline & $\mathrm{HR}(95 \% \mathrm{Cl})$ & $P$-value & $\mathrm{HR}(95 \% \mathrm{Cl})$ & $P$-value \\
\hline Male sex & $0.830(0.304-2.267)$ & 0.715 & $0.560(0.171-1.837)$ & 0.339 \\
\hline Age at diagnosis & 0.987 (0.947-1.028) & 0.506 & 0.994 (0.949-1.042) & 0.813 \\
\hline BMI & $0.989(0.867-1.128)$ & 0.861 & - & \\
\hline Smoking & $0.867(0.255-2.949)$ & 0.820 & - & \\
\hline Disease phenotype B2 & $0.840(0.348-2.029)$ & 0.698 & - & \\
\hline Disease location L1 & 1.555 (0.644-3.753) & 0.328 & - & \\
\hline Perianal lesion present & 1.615 (0.679-3.84) & 0.279 & - & \\
\hline History of surgery & $1.807(0.728-4.483)$ & 0.202 & $3.598(0.827-15.663)$ & 0.088 \\
\hline Failure of EBD & $18.927(7.445-48.116)$ & $0.001^{\mathrm{a}}$ & $17.276(3.546-84.159)$ & $<0.001^{\mathrm{a}}$ \\
\hline Symptomatic bowel obstruction & $2.118(0.624-7.195)$ & 0.230 & - & \\
\hline Serum albumin (mg/dL) & $0.693(0.287-1.674)$ & 0.415 & - & \\
\hline Serum CRP (mg/dL) & $1.287(0.920-1.800)$ & 0.142 & $1.008(0.646-1.572)$ & 0.974 \\
\hline CDAl & $1.011(1.005-1.016)$ & $0.001^{\mathrm{a}}$ & $1.007(1.000-1.014)$ & $0.039^{a}$ \\
\hline Use of thiopurines & $0.535(0.180-1.591)$ & 0.261 & - & \\
\hline Use of anti-TNF- $\alpha$ antibody & $1.622(0.683-3.851)$ & 0.274 & - & \\
\hline Prototype SBE & $1.652(0.677-4.027)$ & 0.270 & - & \\
\hline EBD for multiple strictures & $0.554(0.224-1.374)$ & 0.203 & $0.374(0.111-1.265)$ & 0.114 \\
\hline De novo type of strictures & $1.141(0.383-3.407)$ & 0.814 & - & \\
\hline Long stricture ( $\geq 2 \mathrm{~cm})$ & $11.258(4.109-30.845)$ & $0.001^{\mathrm{a}}$ & $4.737(1.290-17.388)$ & $0.019^{a}$ \\
\hline
\end{tabular}

${ }^{a}$ Significant factor.

B2, stricturing; L1, ileal; EBD, endoscopic balloon dilatation; SBE, single-balloon enteroscopy.

pared with conventional SBE, prototype SBE has significantly improved the depth of insertion (Fig. 3C). A representative case is shown in Fig. 4. Use of the prototype SBE improved the depth of insertion and increased the number of EBDs.

\section{DISCUSSION}

The prototype SBEs with a passive bending mechanism and high transmission insertion tube did not affect long-term EBD outcome, but they did contribute to improving the depth of insertion in the small intestine compared with the conventional SBE. This is the first study to show that prototype SBEs with this mechanism and insertion tube improve insertability in the strictured small intestine.

The EBD success rate in this study was $88.3 \%$, which is consistent with the $62 \%$ to $100 \%$ success rate reported in a pooled analysis and meta-analyses. ${ }^{10,22,23}$ Our 5- and 10-year cumulative surgery-free survival rate of $75.7 \%$ and $72.8 \%$, respectively, was equivalent to the 3 -year rate of $73 \%$ reported by Hirai et al. ${ }^{16}$ and are slightly better than the 1- and 3-year survival rates of $63.1 \%$ and $56.2 \%$, respectively reported by Nishida et al. ${ }^{13}$ At our institution, patients who undergo EBD typically repeat procedures every 1 to 2 years for surveillance and evaluation regardless of the presence or absence of stenotic symptoms. In fact, 23 of the 94 patients (24.4\%) had asymptomatic stenosis. The patients reported by Nishida et al. ${ }^{13}$ had stenotic symptoms, which may be the reason for the differences in results.

In an EBD study involving strictures in both small and large intestine, it was reported that the HR for intestinal resection increased by $8 \%$ when the length of stenosis increased by 1 $\mathrm{cm}^{10}$ In our study, an increase in the length of stenosis in the small intestine by $1 \mathrm{~cm}$ increased intestinal resection by 2.53 times. Our results suggest that increased length of stenosis in the small intestine may require more intestinal resection than increased length of stenosis in the colon.

Multivariate analysis revealed that long stricture $(\geq 2 \mathrm{~cm})$, EBD failure, and elevated CDAI were factors contributing to intestinal resection of small intestinal stricture. Long stricture $(\geq 2$ $\mathrm{cm})^{17}$ and EBD failure 16 have already been reported as factors contributing to intestinal resection. Although elevated CDAI 
Table 3. Clinical Background of the Patients

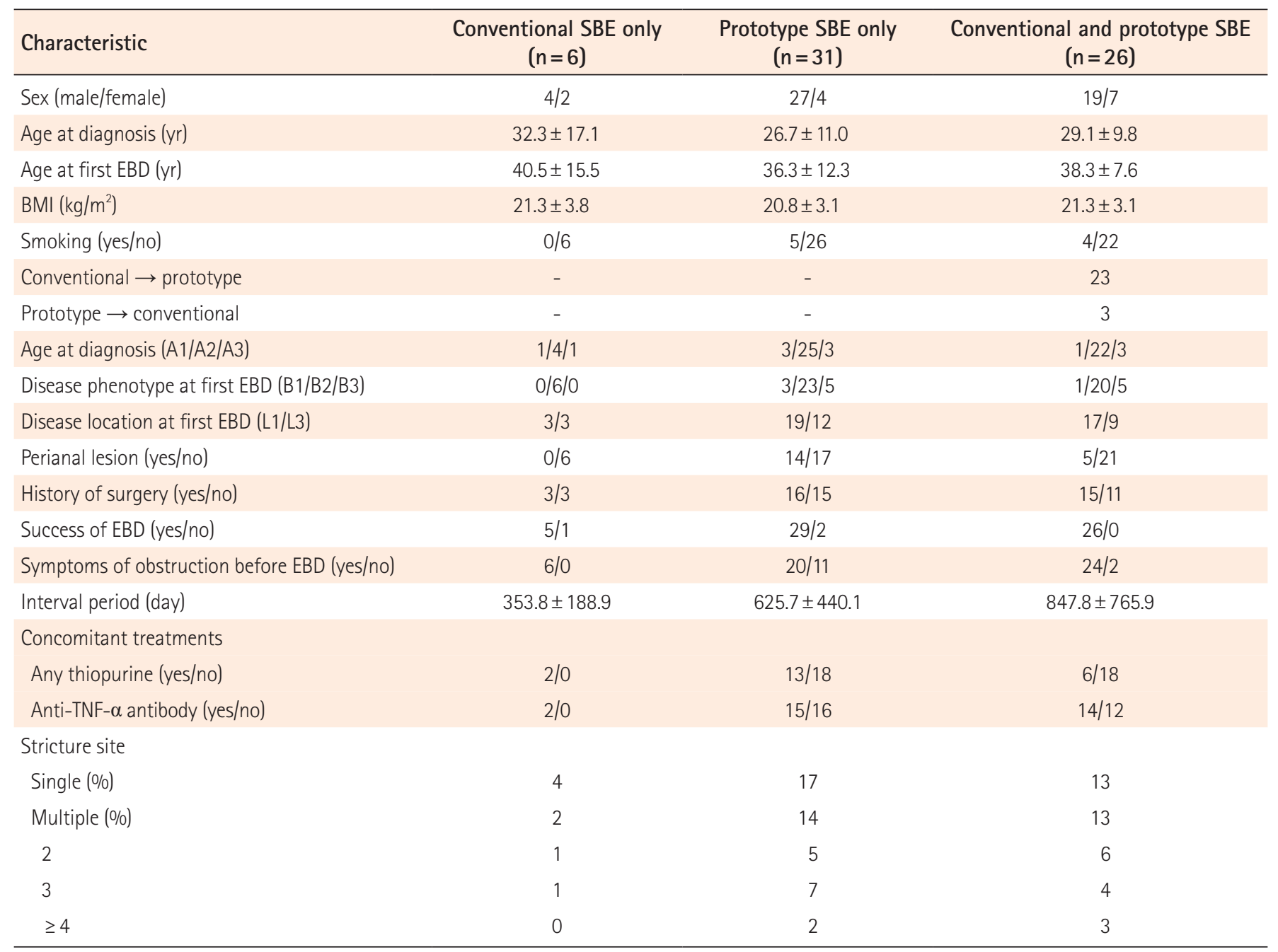

Values are presented as mean \pm SD.

SBE, single-balloon enteroscopy; EBD, endoscopic balloon dilatation; $A 1$, $\leq 16$ years; $A 2,17-40$ years; $A 3$, > 40 years; $B 1$, non-stricturing, nonpenetrating; $B 2$, stricturing; $B 3$, penetrating; L1, ileal; L3, ileocolonic.

has been shown to be associated with intestinal resection in $\mathrm{CD}^{24}$ these cohorts comprised 23\% (20/86) colonic stenosis and 8\% (7/86) upper GI stenosis. Therefore, the novel finding in this study is that the elevated CDAI could be related to intestinal resection even in a cohort limited to the small intestine.

Until now, there have been no reports on achieving deep insertion in the small intestine using a prototype SBE with a passive bending mechanism and high force transmission insertion tube. Although Hosoe et al. ${ }^{19}$ reported that the rate of insertion to the terminal ileum $20 \mathrm{~cm}$ beyond the ileocecal valve within 10 minutes was improved from $86.2 \%$ to $96.8 \%$ by using a prototype SBE in a prospective study, the deep insertability in the small intestine has not been studied. Our findings indicate that the depth of insertion can be significantly improved by using these prototype SBEs in patients with stricturing CD.

The prototype SBEs did not contribute to the cumulative surgery-free survival rate (data not shown). Depth of insertion was increased with the prototype SBEs compared with the conventional SBE, but this difference did not directly improve the approach to stenosis located deep in the small intestine. In fact, the prototype SBEs did not increase the number of EBDs compared with the conventional SBE $(2.1 \pm 1.8$ vs. $1.9 \pm 1.4)$ (Fig. 3I). However, there appeared to be some cases where stenosis could be approached using only a prototype SBE, so further accumulation of cases is necessary in the future.

There are some limitations to this study. First, this is a nonrandomized retrospective study. Therefore, the procedure time during insertion in the small intestine was not recorded. 
A

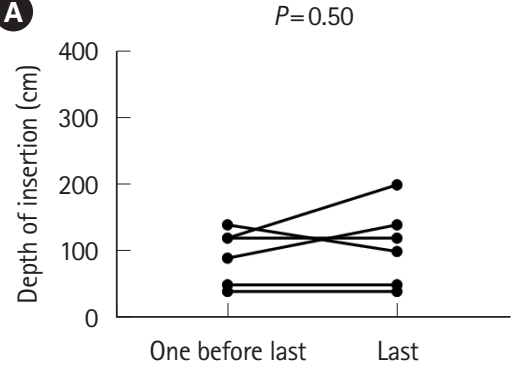

(D)

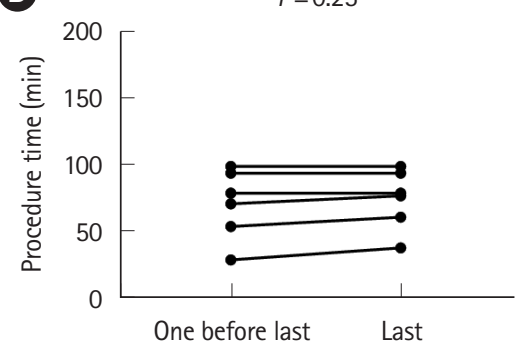

G

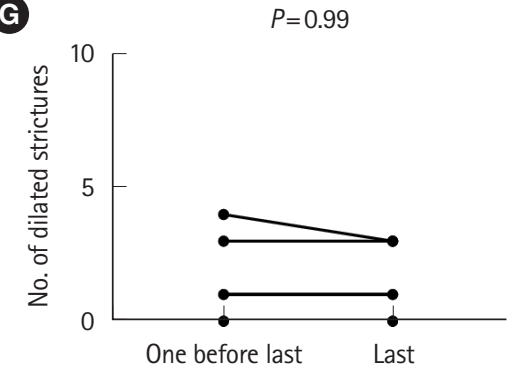

B

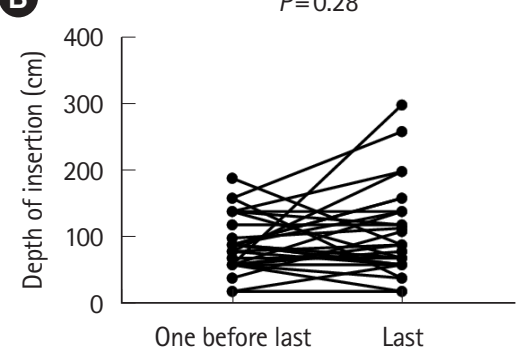

E

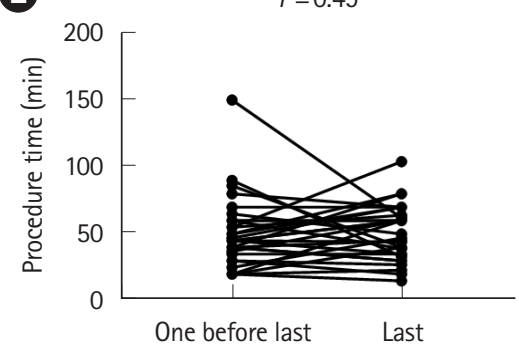

$\boldsymbol{H}$

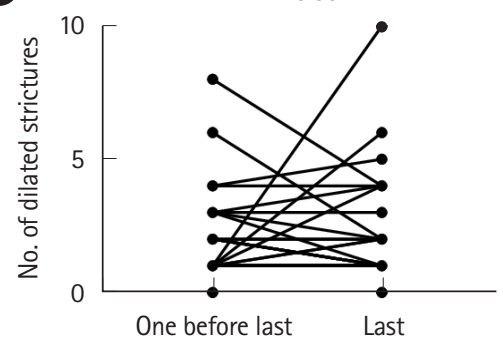

C

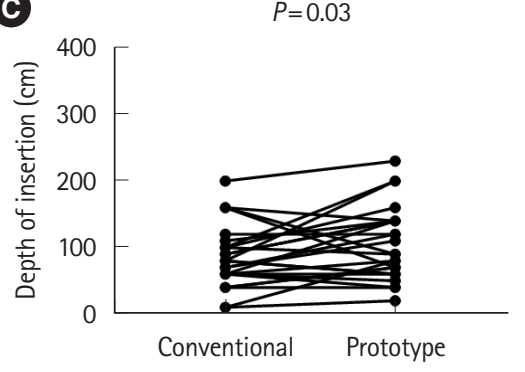

$\boldsymbol{F}$

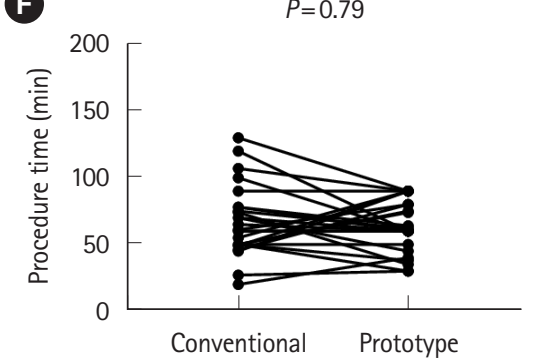

(1)

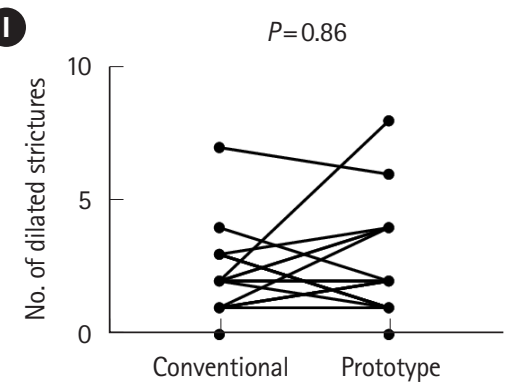

Fig. 3. Insertability and scope used. Patients who underwent single-balloon enteroscopy (SBE) at least twice during the observation period were extracted and divided into 3 groups according to the scope used: $(A, D, G)$ conventional SBE only; $(B, E, H)$ prototype $S B E$ only; and $(C, F, I)$ both conventional and prototype SBEs. For comparison of deep insertability into the small intestine, the 2 most recent procedures were compared in the conventional SBE only group and the prototype SBE only group. The most recent switching opportunity was compared in the group that underwent both conventional and prototype SBE. For each group, depth of insertion $(A-C)$, procedure time (D-F), and number of endoscopic balloon dilatations (G-I) were compared using Wilcoxon's signed-rank test.
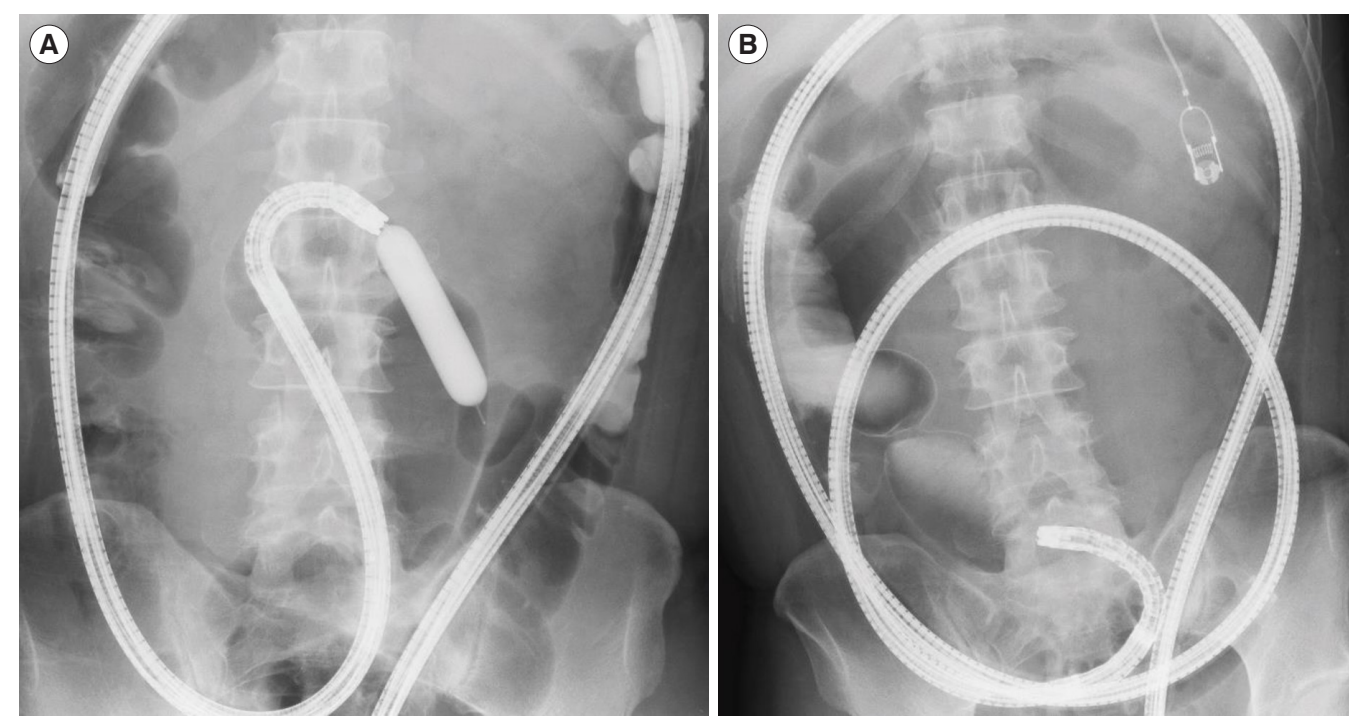

Fig. 4. Representative case. $X-$ ray images at the time of endoscopic balloon dilatation (EBD) at different time points in the same patient. Depth of insertion and the number of EBDs were increased when using single-balloon enteroscopy (SBE) with a passive bending mechanism and high force transmission insertion tube. (A) Conventional SBE: depth of insertion $(70 \mathrm{~cm})$ and number of EBDs $(n=2)$. (B) Prototype SBE: depth of insertion $(120 \mathrm{~cm})$ and number of EBDs $(n=8)$. 
Because the procedure time includes the time of insertion in the large intestine, duration of EBD, and observation period, it was not possible for us to analyze the insertion time in the small intestine. Second, depth of insertion was measured using the method described by May et al., ${ }^{21}$ but this method may be less accurate than the method using crystal violet reported by Takenaka et al. ${ }^{25}$ Third, the timing of the procedures compared to analyze the insertability was different although these procedures were performed in the same patient. Therefore, the condition of the patient may have changed. Generally, the pathology of CD is progressive, so insertion of the enteroscope becomes increasingly difficult as intestinal deformity progresses. It should be noted that the insertability improved even though most of the procedures with a prototype SBE were performed later than those with the conventional SBE.

In conclusion, the prototype SBEs with a passive bending mechanism and high force transmission insertion tube did not improve long-term EBD prognosis, but they did improve the depth of insertion compared with a conventional SBE. In addition, elevated CDAI was identified as a factor contributing to intestinal resection even in a cohort limited to CD patients with strictures in the small intestine.

\section{FINANCIAL SUPPORT}

The authors received no financial support for the research, authorship, and/or publication of this article.

\section{CONFLICT OF INTEREST}

The prototype single-balloon enteroscopes were provided on loan, free of charge, by the Olympus Corporation. No other potential conflict of interest relevant to this article was reported.

\section{AUTHOR CONTRIBUTION}

Conceptualization: Morita Y, Bamba S. Formal analysis: Morita Y, Bamba S. Investigations: all authors. Writing - original draft: Morita Y. Writing - review and editing: Morita Y, Bamba S, Inatomi O, Takahashi K, Imai T. Supervision: Andoh A. Approval of final manuscript: all authors.

\section{ORCID}

Morita Y https://orcid.org/0000-0002-4875-662X

Bamba S https://orcid.org/0000-0002-4108-5894
Inatomi O https://orcid.org/0000-0002-5837-6575

Takahashi K https://orcid.org/0000-0002-3522-9740

Imai T https://orcid.org/0000-0002-9170-4075

Murata M https://orcid.org/0000-0002-4951-0584

Ohno M https://orcid.org/0000-0003-3505-7835

Sasaki M https://orcid.org/0000-0002-0683-1919

Tsujikawa T https://orcid.org/0000-0002-8351-5936

Andoh A https://orcid.org/0000-0001-8533-2669

\section{SUPPLEMENTARY MATERIAL}

Supplementary materials are available at the Intestinal Research website (https://www.irjournal.org).

\section{REFERENCES}

1. Shi HY, Ng SC. The state of the art on treatment of Crohn's disease. J Gastroenterol 2018;53:989-998.

2. Harper PH, Fazio VW, Lavery IC, et al. The long-term outcome in Crohn's disease. Dis Colon Rectum 1987;30:174-179.

3. Munkholm P, Langholz E, Davidsen M, Binder V. Disease activity courses in a regional cohort of Crohn's disease patients. Scand J Gastroenterol 1995;30:699-706.

4. Bernell O, Lapidus A, Hellers G. Risk factors for surgery and postoperative recurrence in Crohn's disease. Ann Surg 2000; 231:38-45.

5. Greenstein AJ, Zhang LP, Miller AT, et al. Relationship of the number of Crohn's strictures and strictureplasties to postoperative recurrence. J Am Coll Surg 2009;208:1065-1070.

6. Limketkai BN, Parian AM, Shah ND, Colombel JF. Short bowel syndrome and intestinal failure in Crohn's disease. Inflamm Bowel Dis 2016;22:1209-1218.

7. Scimeca D, Mocciaro F, Cottone M, et al. Efficacy and safety of endoscopic balloon dilation of symptomatic intestinal Crohn's disease strictures. Dig Liver Dis 2011;43:121-125.

8. Saunders BP, Brown GJ, Lemann M, Rutgeerts P. Balloon dilation of ileocolonic strictures in Crohn's disease. Endoscopy 2004;36:1001-1007.

9. Rieder F, Zimmermann EM, Remzi FH, Sandborn WJ. Crohn's disease complicated by strictures: a systematic review. Gut 2013;62:1072-1084

10. Bettenworth D, Gustavsson A, Atreja A, et al. A pooled analysis of efficacy, safety, and long-term outcome of endoscopic balloon dilation therapy for patients with stricturing Crohn's disease. Inflamm Bowel Dis 2017;23:133-142.

11. Matsuoka K, Kobayashi T, Ueno F, et al. Evidence-based clini- 
cal practice guidelines for inflammatory bowel disease. J Gastroenterol 2018;53:305-353.

12. Lian L, Stocchi L, Remzi FH, Shen B. Comparison of endoscopic dilation vs surgery for anastomotic stricture in patients with Crohn's disease following ileocolonic resection. Clin Gastroenterol Hepatol 2017;15:1226-1231.

13. Nishida Y, Hosomi S, Yamagami H, et al. Analysis of the risk factors of surgery after endoscopic balloon dilation for small intestinal strictures in Crohn's disease using double-balloon endoscopy. Intern Med 2017;56:2245-2252.

14. Sunada K, Shinozaki S, Nagayama M, et al. Long-term outcomes in patients with small intestinal strictures secondary to Crohn's disease after double-balloon endoscopy-assisted balloon dilation. Inflamm Bowel Dis 2016;22:380-386.

15. Hirai F, Beppu T, Sou S, Seki T, Yao K, Matsui T. Endoscopic balloon dilatation using double-balloon endoscopy is a useful and safe treatment for small intestinal strictures in Crohn's disease. Dig Endosc 2010;22:200-204.

16. Hirai F, Beppu T, Takatsu N, et al. Long-term outcome of endoscopic balloon dilation for small bowel strictures in patients with Crohn's disease. Dig Endosc 2014;26:545-551.

17. Okazaki N, Inokuchi T, Hiraoka S, et al. Findings of retrograde contrast study through double-balloon enteroscopy predict the risk of bowel resections in patients with Crohn's disease with small bowel stenosis. Inflamm Bowel Dis 2017;23:20972103

18. Sato K, Ito S, Shigiyama F, et al. A prospective randomized study on the benefits of a new small-caliber colonoscope. En- doscopy 2012;44:746-753.

19. Hosoe N, Ohtsuka K, Endo Y, et al. Insertability comparison of passive bending single-balloon prototype versus standard single-balloon enteroscopy: a multicenter randomized nonblinded trial. Endosc Int Open 2018;6:E1184-E1189.

20. Satsangi J, Silverberg MS, Vermeire S, Colombel JF. The Montreal classification of inflammatory bowel disease: controversies, consensus, and implications. Gut 2006;55:749-753.

21. May A, Nachbar L, Schneider M, Neumann M, Ell C. Pushand-pull enteroscopy using the double-balloon technique: method of assessing depth of insertion and training of the enteroscopy technique using the Erlangen Endo-Trainer. Endoscopy 2005;37:66-70.

22. Morar PS, Faiz O, Warusavitarne J, et al. Systematic review with meta-analysis: endoscopic balloon dilatation for Crohn's disease strictures. Aliment Pharmacol Ther 2015;42:11371148.

23. Navaneethan U, Lourdusamy V, Njei B, Shen B. Endoscopic balloon dilation in the management of strictures in Crohn's disease: a systematic review and meta-analysis of non-randomized trials. Surg Endosc 2016;30:5434-5443.

24. Mao R, Chen BL, He Y, Cui Y, Zeng ZR, Chen MH. Factors associated with progression to surgery in Crohn's disease patients with endoscopic stricture. Endoscopy 2014;46:956-962.

25. Takenaka K, Ohtsuka K, Kitazume Y, et al. Comparison of magnetic resonance and balloon enteroscopic examination of the small intestine in patients with Crohn's disease. Gastroenterology 2014;147:334-342. 
See "Prototype single-balloon enteroscopy with passive bending and high force transmission improves depth of insertion in the small intestine" on page 229-237.

Supplementary Table 1. Enteroscope Specifications

\begin{tabular}{|c|c|c|c|c|}
\hline \multirow{2}{*}{ Specification } & \multicolumn{3}{|c|}{ Prototype SBE } & \multirow{2}{*}{$\begin{array}{c}\text { Conventional SBE } \\
\text { SIF-0260 }\end{array}$} \\
\hline & SIF-Y0013 & SIF-Y0002 & SIF-Y0007 & \\
\hline \multicolumn{5}{|l|}{ Optical system } \\
\hline Field of view $\left({ }^{\circ}\right)$ & 140 & 140 & Wide: 140, tele: 60 & 140 \\
\hline Direction of view & Forward viewing & Forward viewing & Forward viewing & Forward viewing \\
\hline Depth of field (mm) & $3-100$ & $3-100$ & Wide: $7-100$, tele: $2-3.5$ & $5-100$ \\
\hline Magnification & - & - & $\times 80$ & - \\
\hline \multicolumn{5}{|l|}{ Distal end } \\
\hline Outer diameter (mm) & 9.2 & 9.2 & 9.9 & 9.2 \\
\hline \multicolumn{5}{|l|}{ Insertion tube } \\
\hline Outer diameter (mm) & 9.2 & 9.2 & 9.2 & 9.2 \\
\hline High force transmission & Yes & Yes & Yes & No \\
\hline \multicolumn{5}{|l|}{ Bending section } \\
\hline Angulation range $\left({ }^{\circ}, U / D / R / L\right)$ & $180 / 180 / 160 / 160$ & 180/180/160/160 & $180 / 180 / 160 / 160$ & 180/180/160/160 \\
\hline Passive bending & Yes & Yes & Yes & No \\
\hline Working length (mm) & 2,000 & 2,000 & 2,000 & 2,000 \\
\hline Total length (mm) & 2,345 & 2,345 & 2,345 & 2,345 \\
\hline \multicolumn{5}{|l|}{ Instrumental channel } \\
\hline Inner diameter (mm) & 3.2 & 2.8 & 2.8 & 2.8 \\
\hline Overtube & ST-SB1 & ST-SB1 & ST-SB1 & ST-SB1 \\
\hline
\end{tabular}

SBE, single-balloon enteroscopy; U, up; D, down; R, right; L, left. 